\title{
Central Projections of Sensory Neurons in the Drosophila Embryo Correlate with Sensory Modality, Soma Position, and Proneural Gene Function
}

\author{
David J. Merritt and Paul M. Whitington \\ Department of Zoology, University of New England, Armidale, New South Wales 2351, Australia
}

The peripheral nervous system (PNS) of the Drosophila embryo is especially suited for investigating the specification of neuronal identity: the PNS consists of a relatively simple but diverse set of individually identified sensory neurons; mutants, Including embryonic lethals, can be readily generated and analyzed; and axon growth can potentially be followed from the earliest stages. We have developed a staining method to reveal the central projections of the full set of sensory neurons in the preterminal abdominal segments of the embryo. The sensory neurons exhibit modality-specific axonal projections in the CNS. The axons of external sense (es) organ neurons, primarily tactile in function, are restricted to a particular region within each neuromere and exhibit a somatotopic mapping within the CNS. The axons of stretch-receptive chordotonal (ch) organs project into a discrete longitudinal fascicle. Sensory neurons with multiple-branched dendrites (md neurons) project into a separate fascicle. A small number of md neurons have distinctive dorsal-projecting axonal processes in the CNS. A classification of sensory neurons based on their axon morphology correlates closely with the identity of the proneural gene responsible for their generation, suggesting that proneural genes play a central role in determining neuronal identity in the PNS of the embryo.

[Key words: sensory afferent, neural development, proneural genes, topographic maps, insect, sense organs, neuronal identity]

The Drosophila embryo is an especially attractive model system for investigating the cellular and molecular basis of neuronal determination and morphogenesis. First, the entire embryonic and larval PNS has been described at the level of individually identified cells (Ghysen et al., 1986; Bodmer and Jan, 1987; Bodmer et al., 1989). There is a modest population of approximately 40 sense organs (SOs) per hemisegment, which fall into two classes: type I SOs have neurons with monopolar, ciliated dendrites and type II SOs have neurons with nonciliated, branched dendrites (md organs) (Bodmer and Jan, 1987). Type I SOs include the cxternal, touch-sensitive sense (es) organs, and the internal, proprioceptive chordotonal (ch) organs which main-

\footnotetext{
Received June 7, 1994; revised Aug. 11, 1994; accepted Aug. 18, 1994.

We thank Dr. S. Cairns for help with the statistical analysis, Dr. E. Ball for supplying anti-HRP-stained embryos, and Dr. C. Goodman for supplying $22 \mathrm{C} 10$ antibody. This work was supported by an Australian Research Council grant to P.M.W.

Correspondence should be addressed to Dr. D. J. Merritt at the above address. Copyright $(\mathcal{O} 1995$ Society for Neuroscience $0270-6474 / 95 / 151755-13 \$ 05.00 / 0$
}

tain an indirect attachment to the cuticle (Hartenstein, 1988). Second, mutations which result in an embryonic lethal phenotype, and which therefore evade recognition and analysis at later developmental stages, are open to investigation (see reviews by Jan and Jan, 1990; Vaessin et al., 1990; Ghysen and DamblyChaudière, 1992, 1993; Ghysen et al., 1993).

In the early embryo, the potential to form sensory neurons comes about through expression of the proneural genes in segmentally reiterated patches of ectoderm. There are two known categories of proneural genes active in the embryonic PNS, the achaete-scute complex (ASC) and atonal (Ghysen and DamblyChaudière, 1988; Jarman et al., 1993). ASC-expressing ectoderm produces all external sense (es) organs and a subset of multiple dendrite (md) neurons, while atonal-expressing ectoderm produces all chordotonal (ch) organs and most of the remaining $\mathrm{md}$ neurons (Dambly-Chaudière and Ghysen, 1987; Jarman et al., 1993).

Proneural gene action causes an individual cell within each ectodermal patch to become a sense organ precursor (SOP). This cell inhibits surrounding cells in the proneural patch from progressing down the neural pathway and the resulting SOP either undergoes further divisions to produce both the sensory neuron and its associated support cells (for type I organs) or differentiates directly into a sensory neuron (for type II organs) (Bodmer et al., 1989).

A further class of genes, the identity selector genes, is currently thought to determine the specific mode of division of the SOP, as well as the identities of its progeny. The gene cut is one example of this class of gene: in embryos lacking cut activity es organs are replaced by ch organs (Bodmer et al., 1987). Another is the pox-neuro gene, responsible for the differentiation of es SOPs into monoinnervated or polyinnervated SOs (Dambly-Chaudière et al., 1992). The BarHI and BarH2 genes specify whether an es organ will have a cuticular hair or papilla (Higashijima et al., 1992). However, whether SO identity is solely specified by this class of genes is open to question.

One of the most important expressions of a sense organ's identity is the pattern of axonal projections made by the sensory neuron in the CNS: the neuron can make synaptic contact only with central neurons that extend processes into the region covered by its axonal arborizations. Studies of the central projections of sensory neurons in adult insects, including Drosophila, have revealed some of the characteristics of the projections of sensory neurons in the CNS.

There is a relationship between the pattern of central projections and the type or modality of sensory neuron. Tactile axons project into a distinctive ventral neuropil, the ventral association 
center (VAC), found in a wide range of insects including grasshoppers, moths, crickets, and flies (Johnson and Murphey, 1985; Murphey et al., 1985; Pflüger et al., 1988). Proprioceptive sense organs send their axons into an intermediate or dorsal neuropil region of each neuromere (Murphey et al., 1985; Pflüger et al., 1988; Murphey et al., 1989b; Merrill and Murphey, 1992), while gustatory neurons are partitioned from the tactile afferents (Murphey et al., 1989b; Nottebohm et al., 1992).

The position of the SO on the body can influence its axonal projection: the axon terminals of touch-sensitive neurons are arranged in relation to one another such that their relative positions at the surface are maintained within the VAC in the CNS (Ghysen, 1980; Bacon and Murphey, 1984; Levine et al., 1985; Kent and Levine, 1988; Peterson and Weeks, 1988; Murphey et al., 1989a,b; Newland, 1991).

In contrast to this large body of information for adult Drosophila and other insects, little is known about the central projections of sensory neurons in the embryo or larval stages of Drosophila, as the methods used to reveal adult projections are not applicable to these earlier developmental stages. We have recently developed techniques for staining single sensory neurons in the Drosophila embryo with fluorescent dyes to reveal their central projections. We found that two types of SOs, the external sense (es) organs and chordotonal (ch) organs, have different projections (Merritt et al., 1993). In the only other report of sensory axon morphology in the embryo, subsets of sensory axons were stained using "enhancer trap" markers carrying $\beta$-galactosidase linked to kinesin, which transports the marker enzyme to the axonal extremities (Giniger et al,, 1993). In one transformant line, all ch neurons express the marker enzyme, revealing an axon pattern consistent with the picture obtained from staining individual ch neurons.

In this study, we sought to extend our knowledge of the central projections of sensory neurons in the wild-type Drosophila embryo. We report here the axon projections from the complete population of sensory neurons in abdominal segments Al-7. They fall into three major categories: es axon projections, ch projections, and md-da projections, each identifiable by their partitioning into discrete regions of the neuropile in each neuromere. Among the es neurons, there is evidence of a somatotopic mapping of axons into the CNS in accord with the position of the SO at the body wall. Some md neurons have distinctive axonal projections which do not fall into the typical md-da group. We have observed a strong correlation between the projection pattern of a given class of sensory neuron and the identity of the proneural gene that is responsible for its generation, indicating that proneural gene activity may be an important determinant of neuronal identity.

\section{Materials and Methods}

Embryo preparation. Eggs at stage late 16 or stage 17 (Campos-Ortega and Hartenstein, 1985) were dechorionated in bleach and the embryos squeezed out of the vitelline membrane under Ringer solution after cutting off the anterior end. Embryos were attached under Ringer solution to glass slides precoated with $10 \%$ poly-L-lysine (Sigma, St. Louis, $\mathrm{MO}$ ), and the sensory neurons viewed using Nomarski optics and a $100 \times$ watcr-immersion objective.

Neuronal staining. Two staining techniques were used, intracellular injection of Lucifer yellow CH (Ly; Sigma) or the lipid-soluble carbocyanine dye $1,1^{\prime}$-dioctadecyl-3,3,3',3'-tetramethyl indocarbocyanine perchlorate (diI; Molecular Probes, Eugene, OR) into the cell body. Injection and immunocytochemical staining of Ly has been described by Merritt et al. (1993). For diI staining, the tips of microelectrodes were filled with an ethanolic solution of dye and the shaft filled with
$0.1 \mathrm{M} \mathrm{LiCl}$. The tip of the microelectrode was brought into contact with a sensory neuron cell body and depolarizing current applied for up to $1 \mathrm{~min}$. Embryos were dissected dorsally to expose the CNS, fixed, and washed in phosphate-buffered saline. Because of the relatively slow diffusion rate of dil in axonal membranes, the end processes of cells with longer axons did not reach full staining intensity for 2-3 hr. Preparations were photoconverted in the presence of $0.2 \%$ diaminobenzidene (DAB) to give a permanent dark reaction product (Sandell and Masland, 1988) and drawn using a camera lucida. Specimens were washed in PBS and mounted in $75 \%$ glycerol or Faure's medium (Wieschaus and Nüsslein-Volhard, 1986).

Recording neuronal morphology. Arborizations of sensory neurons were drawn with the aid of a camera lucida and digitized with an Apple scanner. To compensate for tissue shrinkage, drawings were scaled to fit a CNS template using the positions of the anterior and posterior commissures, the anterior and posterior fascicles, and the width of the CNS as landmarks, using CANVAS software (Deneba Software, Miami) on a Macintosh computer. Selected specimens were viewed using a Pulnix video camera (Eugene, OR) mounted on a Zeiss Axioskop and a series of focal planes digitized with a Neotech (Eastleigh, Hampshire, UK) or PixelBuffer (Perceptics, Knoxville) image grabber. Montages of axonal projections were made by collating in-focus regions onto a single plane using the NIH-IMAGE program (written by W. Rasband, available by anonymous ftp from zippy.nimh.nih.gov). Stcrco pairs were generated using the "projection" feature: focal planes were stored as a stack, their pixel values inverted, and brightest-point projections through the stack were made at two rotation angles, providing left- and right-eye images which were reinverted to restore the original pixel values.

22 C10 antibody staining. Dechorionated embryos were fixed by shaking in a mixture of heptane and $3.4 \%$ formaldehyde. After removal of the fixative, embryos were devitellinized by shaking in a methanolheptane mixture, washed in phosphate-buffered saline (PBS), and blocked in PBS with $0.4 \%$ Triton X-100, $0.25 \%$ bovine serum albumin, and $2 \%$ normal goat serum (PBT-NGS) for $1 \mathrm{hr}$. They were incubated overnight at $5^{\circ} \mathrm{C}$ in a $1: 7$ dilution of $22 \mathrm{C} 10$ antibody (Fujita et al., 1982; courtesy of Dr. C. Goodman, Howard Hughes Medical Institute, University of California at Berkeley, CA) in PBT-NGS, washed, and incubated for $3 \mathrm{hr}$ at room temperature in a 1:50 dilution of HRP-conjugated sheep anti-mouse igG (Amersham Australia, Sydney) in PBT-NGS. The secondary antibody was visualized by the DAB reaction with $0.04 \%$ nickel chloride in a $0.025 \%$ DAB solution to give a blue reaction product.

\section{Results}

The abdominal sense organ population of the embryo In the Drosophila embryo, abdominal segments 1-7 (A1-7) have the same pattern of SOs (Fig. 1). There are 40 sense organs with 42 sensory neurons in each hemisegment, all of which have been classified according to the nature of the dendritic processes of their neurons. They are the external sense (es) organs, chordotonal organs (ch), and multiple-dendrite (md) sense organs (Ghysen et al., 1986; Bodmer and Jan, 1987; Bodmer et al., 1989). Within each thoracic and abdominal segment, SOs are clustered into four groups: the dorsal, lateral, ventral', and ventral groups (Ghysen et al., 1986). Axons from the ventral and ventral' sensory neurons run in the segmental nerve, entering the CNS through the posterior fascicle of the homotopic neuromere, while all lateral and dorsal sensory axons run in the intersegmental nerve, entering through the anterior fascicle (Campos-Ortega and Hartenstein, 1985; Hartenstein, 1988). The neuromeres form a chain without obvious discontinuities between segments (Figs. 2, 3). A pair of longitudinal connectives on either side of the midline is joined by an anterior and posterior commissure within each neuromere.

es organs are innervated by one or two neurons with monopolar dendrites (Fig. 2A). The dendrite takes a curved path from the cell body to the associated external cuticular structure such as a hair, a papilla, or basiconic peg (Dambly-Chaudière and Ghysen, 1986). The single-neuron es organs are most likely 


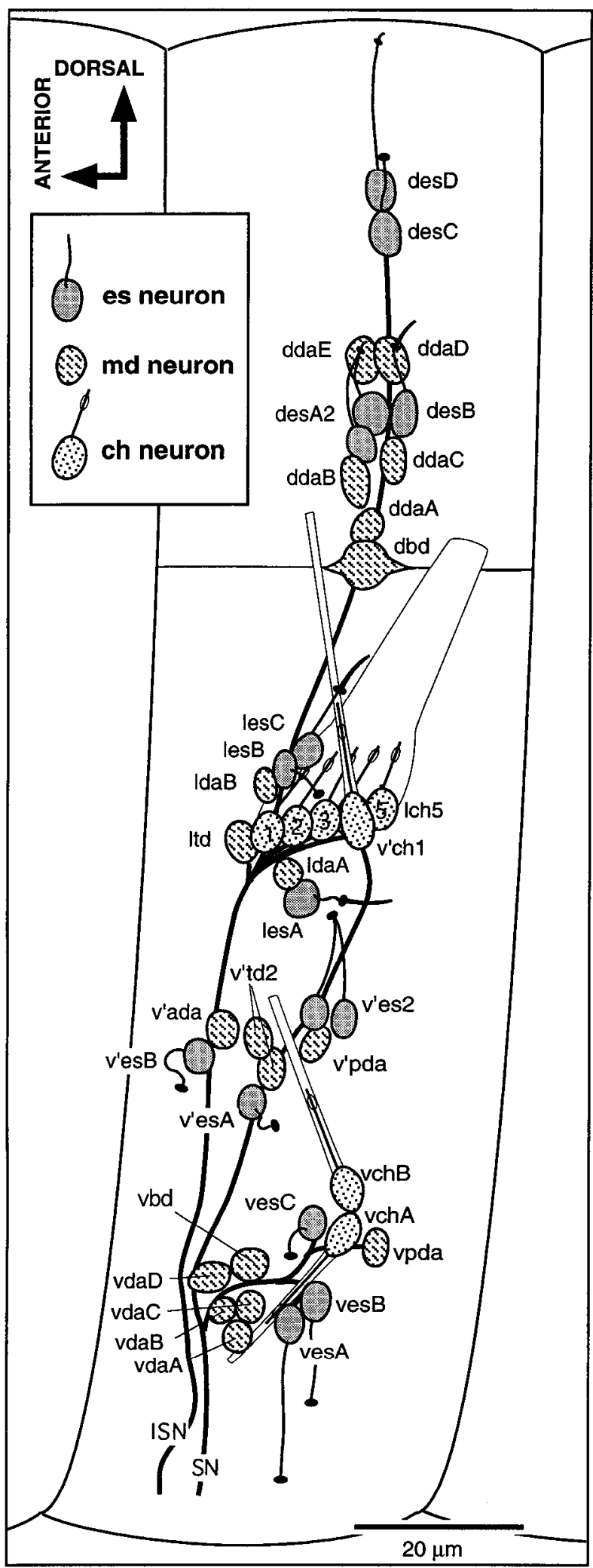

Figure 1. The distribution of sensory neurons in an abdominal segment representative of A1-7. There are 42 neurons per side. External sensory (es) neurons have monopolar dendrites which attach to a cuticular structure such as a hair or peg. Two es organs- $v^{\prime} e s 2$ and desA2are innervated by two neurons. Chordotonal (ch) neurons also have monopolar dendrites although they are not associated with the cuticle and are surrounded by a distinctive scolopale structure. They are present as individuals or clustered in a group of five neurons $(l c h 5)$. Multipolar dendrite $(m d)$ neurons have branched, multiple dendrites. The axons mechanosensory in function (Hartenstein, 1988). In A1-7, two SOs within each hemisegment are innervated by two neurons (Dambly-Chaudière and Ghysen, 1986). Their function is unknown. All es organs appear to have three support cells associated with the neuron at the periphery (Hartenstein, 1988).

ch organs are proprioceptors, responsive to stretching between proximal and distal attachment points to the epidermis. A ch organ comprises a neuron and three support cells. The monopolar dendrite is straight (Fig. 2B) with no attachment to external cuticular features. In A1-7, five aligned ch organs form the pentascolopidial organ and three single ch organs lie in the lateral to ventral region.

md organs have neurons with multiple-branched dendrites. Unlike es and ch organs they do not have support cells, with one possible exception (Bodmer et al., 1989). They have becn subdivided by Bodmer and Jan (1987) into three categories: those with extensive subepidermal dendritic arbors (md-da; Fig. $2 C$ ), those whose dendrites are associated with trachea (md-td), and those with bipolar dendrites (md-bd; Fig. 2D). According to Bodmer and Jan (1987) there are 14 da neurons, 3 td neurons, and 3 bd neurons per hemisegment in Al-7.

\section{The central projections of abdominal sensory neurons}

We find that the individual sensory neurons of A1-7 have characteristic axonal projections: the same sensory neuron in different animals has a similar axon morphology. However, there is a degree of variability in the projection of each neuron. To give an impression of the variability encountered, the axon projections of sensory neurons from several individuals are shown superimposed in Figures 4-7 and 9. Furthermore, the axons of $\mathrm{cs}$, ch, and md-da ncurons havc arborization patterns with groupspecific features, most notably a tendency to be restricted to a particular region within their neuromere. The region occupied by typical sensory neurons of each group is shown in Figure 3. Two representatives are shown of each: a ventral neuron whose axon enters the CNS through the posterior fascicle, and a lateral or dorsal neuron whose axon enters through the anterior fascicle. Several md neurons do not fit into any of these three categories-their axon projections are described in the final section of the Results.

\section{ch neurons}

ch neurons characteristically have lengthy rostrocaudal axonal processes which lie within a central fascicle in the connective (Figs. 3,4 ). They display a higher degree of subbranching than

$\leftarrow$

from all neurons in the ventral and ventral' regions (their designations begin with $v$ or $v^{\prime}$ ) run in the segmental nerve $(S N)$ toward the CNS, where they enter via the posterior fascicle. The axons of lateral and dorsal neurons ( $l$ or $d$ designations) run in the intersegmental nerve $(I S N)$ to enter the CNS via the posterior root of the anterior fascicle. Neurons are named according to Ghysen et al. (1986) for es and ch organs and Bodmer et al. (1989) for md neurons. We have modified the nomenclature for md neurons in two respects. The vmd5 group of Bodmer et al. (1989) comprises four md-da neurons and a single bipolar dendrite neuron. We have termed the bipolar dendrite neuron vbd, and the adjacent md neurons vdaA-D from ventral to dorsal, respectively. Similarly, the da neurons in the dorsal cluster have been named the dda group. Their individual designations are arbitrary as they could not be individually distinguished. The exact number of md neurons in this group is uncertain: there may be five (Bodmer and Jan, 1987; DamblyChaudière and Ghysen, 1987; Jarman et al., 1993), or six (Bodmer et al., 1989). 

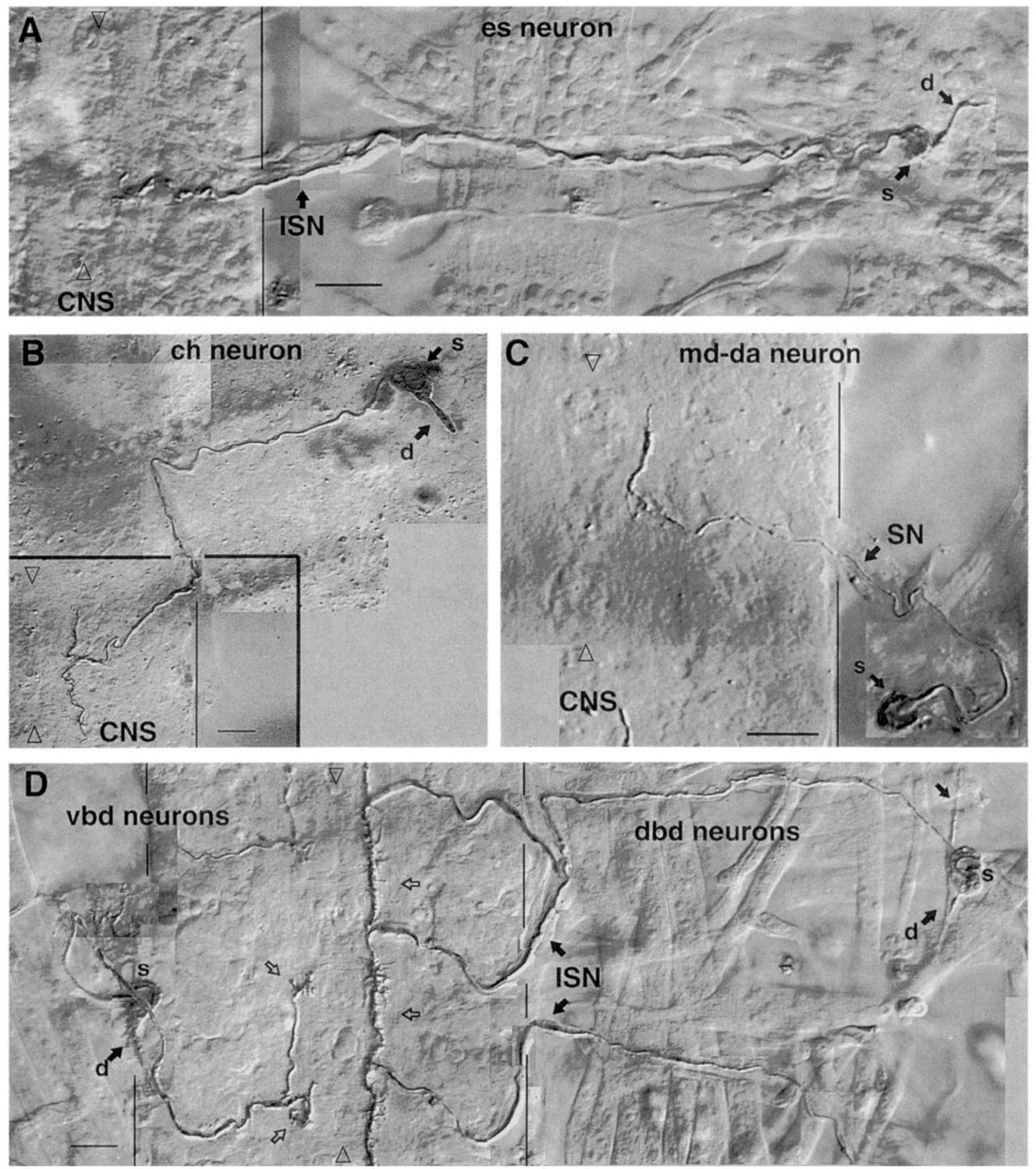

Figure 2. The full neuronal morphology of representative sensory neurons of the embryo. Neurons were stained by application of diI and photoconverted to give a dark reaction product. These composite images were made by collating in-focus regions of the neurons onto single frames and combining several frames to form a montage. In each panel, anterior is to the top and the midline of the CNS is indicated by open arrowheads. The lateral edges of the CNS are shown by vertical bars. A. The neuron of the es organ, lesC. The soma $(s)$, lying beneath the epidermis, has a single curved dendrite $(d$, arrow) and an axon extending into the CNS through the intersegmental nerve (ISN) where it branches in the longitudinal connective. $B$, A ch organ neuron, lch5-2. The dendrite $(d$, arrow) is straight in comparison with the curved es dendrites and in the CNS the axon projects anteriorly and posteriorly. The box encloses the region in which the CNS is in focus. $C$, An md-da neuron, vdaA. The axon extends anteriorly in the longitudinal connective. The dendrites arising from the soma $(s)$ are not visible in this specimen. This axon enters the CNS through the segmental nerve $(S N)$. D. Two bipolar dendrite neurons, vbd and dbd. The soma $(s)$, dendrites $(d)$, and full axonal process of a vbd neuron are shown lower left, in abdominal neuromere 5. Above is the less intensely stained central projection of a vbd neuron in the next anterior segment but one, in abdominal neuromere 3. Its soma is not shown. Characteristic dorsal-running vbd axonal processes within the longitudinal connective 


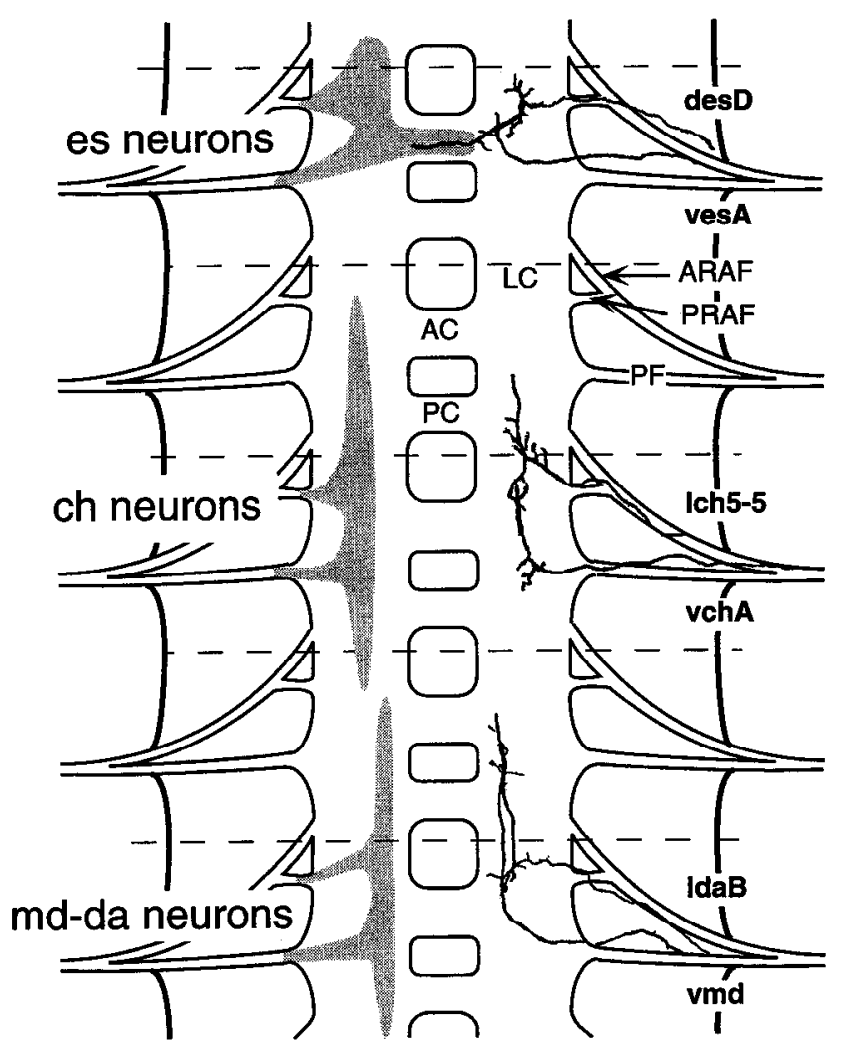

Figure 3. A summary of the projection regions of the three major subtypes of sensory projections. They are es type, ch type, and"md-da type, together accounting for 37 of the 42 neurons of an abdominal hemisegment. These groups are based on the type of axonal branching and arborization distribution in the neuromere. On the left, the region occupied by axons of a particular group is stippled. On the right, two individual axons are shown, one arising from a dorsal sensory neuron and one ventral, showing the effect of entering the CNS through different nerve roots. The $\mathrm{ch}$ and $\mathrm{md}$-da neurons lie in discrete longitudinal fascicles within the connective. The es neurons tend to be more highly branched and, as a group, are not restricted to longitudinal tracts. Putative segment boundaries in the CNS are shown by dotted lines. Anterior is $u p$. $L C$, longitudinal connective; $A C$ and $P C$, anterior and posterior commissures; $A R A F, P R A F$, anterior and posterior roots of the anterior fascicles; $P F$, posterior fascicle.

the md-da neurons, which also have long processes (sec bclow) and occupy a different region within the longitudinal connective to these neurons (Fig. 3). Their position was quantified by expressing the location of the main rostrocaudal process as a fraction of the connective width: lateralmost having a value of 0 and medialmost 1.0. Representatives of all ch neurons in A 1-7 were included in the sample $(n=38)$. A mean $( \pm 1 \mathrm{SD})$ mediolateral (ML) index of $0.48 \pm 0.09$ resulted, indicating that the processes lie close to the middle of each connective. The axons of ventral group ch neurons- $\mathrm{vchA}, \mathrm{vchB}$, and $\mathrm{v}^{\prime} \mathrm{ch} 1-$ have similar central projections, running anteriorly in the middle of the longitudinal connective and entering the CNS through the posterior fascicle (Merritt et al., 1993).

The axons arising from the pentascolopidial organ in the lateral group enter the CNS through the anterior fascicle and pro- ject in both anterior and posterior directions on entering the CNS (Fig. 4). Two of the lch5 neurons, namely lch5-2 and 5-4, have extensive rostrocaudal branches which may extend into neighboring neuromeres, while the others-Ich5-1, 5-3, and 5-4 have more restricted processes. Despite the differences in length of the rostrocaudal projections, the processes of the individual neurons of the pentascolopidial organ overlap considerably in the CNS. Furthermore, the projections of the lch5 neurons overlap considerably with $v$ and $v^{\prime}$ ch axons (Fig. 4). This was confirmed by simultaneous staining of a ventral ch neuron and a neuron from the pentascolopidial organ (data not shown).

\section{es neurons}

The axonal processes of es neurons branch in the CNS in the anterior half of their homotopic neuromere. Some projections extend into the next anterior neuromere (Fig. 3). The highly branched processes of single neurons can cover a wide mediolateral region within the neuromere (Fig. 5), some individuals extending contralaterally. For this reason we did not determine an ML index for this group. In contrast, ch and md-da axons tend to be restricted in the mediolateral axis and extend further in the rostrocaudal axis (Fig. 3).

The projection region of es axons within the CNS appears to be correlated with the position of the SO on the body wall, most obviously in the case of the ventral sensory neurons. A progressive posterior to anterior shift in arbor distribution within the neuromere corresponds with a ventral to dorsal shift in location of the neuron on the body wall.

Ventral es. Within the CNS, the ves and v'es axons extend either medially or anteriorly (Fig. 5). Both vesA and vesB axons generally have a medial-running branch. For vesA, the most ventral SO in A1-7, five of eight individuals stained had a process crossing the midline in the anterior commissure; for vesB, two of five cross the midline. The central arbor of vesC, whose cell body lies more dorsal, did not cross the midline in any of the eight individuals stained. Similarly, the axons of v'esA, v'esB, and v'es 2 extend anteriorly rather than medially (Fig. 5).

The v'es2 SO is unusual in that it is dually innervated. However, the central projections of both neurons do not appear to be markedly different from adjacent es neurons. Simultaneous staining of both neurons of a single $v^{\prime}$ es $2 \mathrm{SO}$ showed no obvious difference between the two (data not shown).

Lateral es. Whereas the ventral es axons enter the CNS through the posterior fascicle, the axons of les neurons run in the intersegmental nerve, entering the CNS through the anterior fascicle. There is a corresponding change in the shape of the arborization within the CNS. In lesA, lesB, and lesC the axonal arborizations lie predominantly posterior to the point of entry of the axon in the CNS. However, there is a high degree of overlap with the anterior part of the projections of the $v^{\prime}$ es neurons.

Dorsal es. The axonal projections from desA2 and desB are presented together in Figure 5. These three neurons could not be reliably identified as individuals because their somata lie very close together. In the CNS their axons branch diffusely over a restricted region in the rostrocaudal axis. $\operatorname{des} C$ and $D$ are the most dorsal es organs in A1-7. desC shows a similar projection

$\leftarrow$

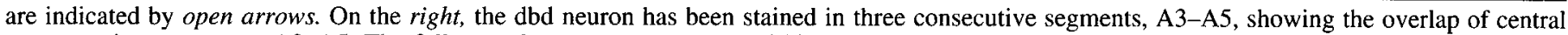

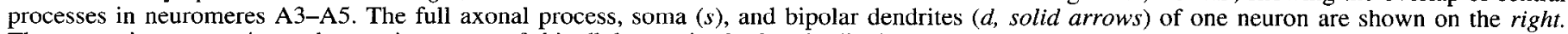
The approximate anterior and posterior extent of this dbd axon in the longitudinal connective is shown by open arrows. Scale bar, $10 \mu \mathrm{m}$. 
Figure 4. The central axonal projections of chordotonal $(c h)$ sensory neurons in the abdominal segments of the embryo. The locations of the ch neurons are shown in the central panel (modified from Fig. 1: dorsal is up and anterior to the left ) and the axonal projections are shown in individual panels (modified from Fig. 2: anterior is up). Within each panel, the left side shows the projections of axons from different individuals superimposed using landmarks in the CNS for alignment. Opposite, a single representative projection is shown. The vch and v'ch axons enter the CNS through the posterior fascicle while the 1ch5 neurons enter via the posterior root of the anterior fascicle.
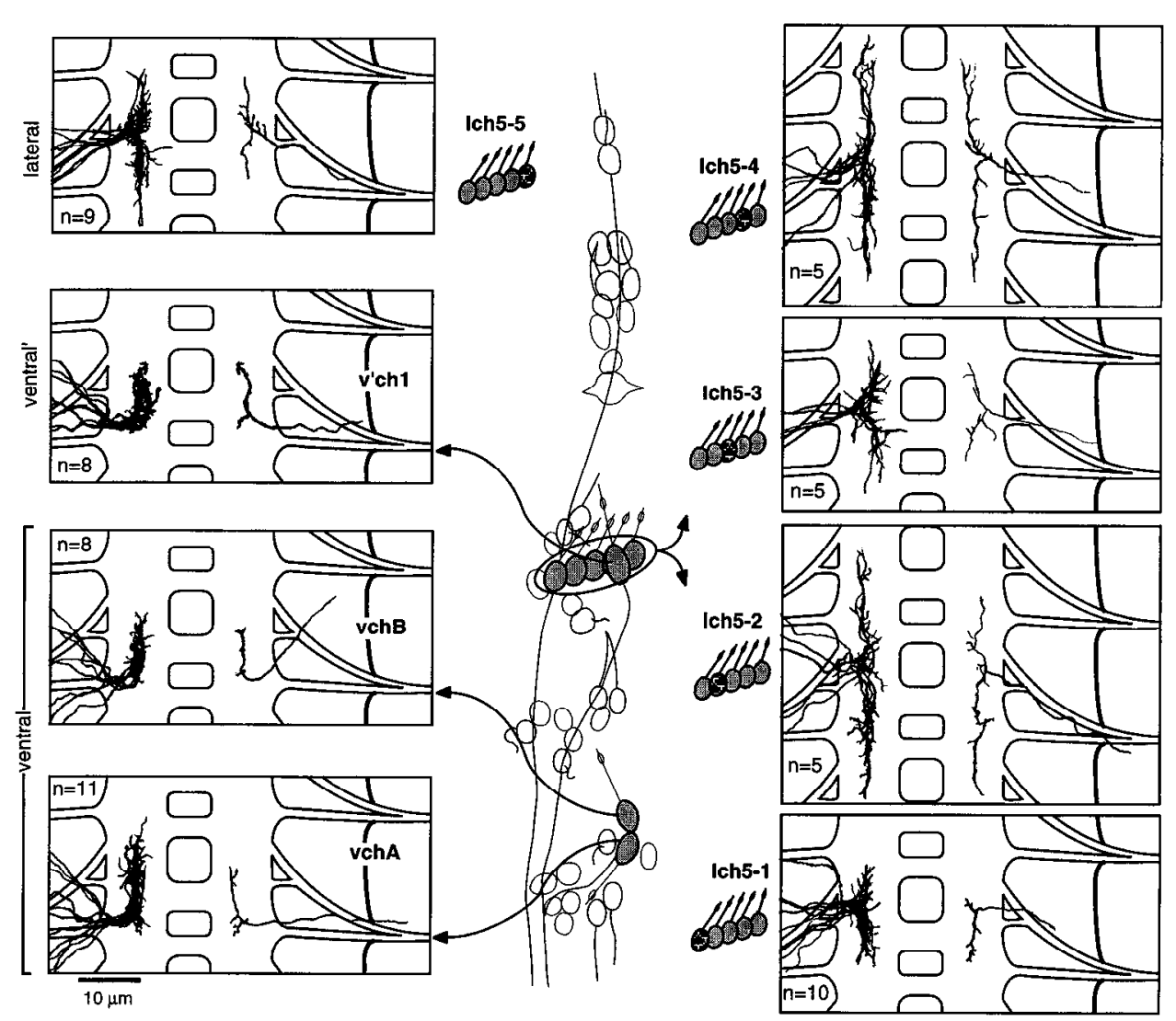

Figure 5. The central axonal projections of es-type sensory neurons in the abdominal segments of the embryo. The positions of the es neurons on the body wall are shown in the central panel (modified from Fig. 1: dorsal is up and anterior to the left), the axonal projections are shown in individual panels (modified from Fig. 2: anterior is $u p$ ), and the type of cuticular structure (hair or papilla), named according to Dambly-Chaudière and Ghysen (1986), shown in the inserts. In cach panel, one side shows the projections of axons from different individuals superimposed using landmarks in the CNS for alignment. Opposite, a single representative projection is shown. The panels are arranged to reflect the dorsal-ventral location of the parent sensory neuron: the axons of the ventralmost neurons are shown in the bottom left column and the dorsalmost in the top right column. Note that at the transition between the ventral' and lateral neuronal locations (top left column to bottom right column), the axons changc their pathway into the CNS, entering via the posterior root of the anterior fascicle rather than the posterior fascicle.

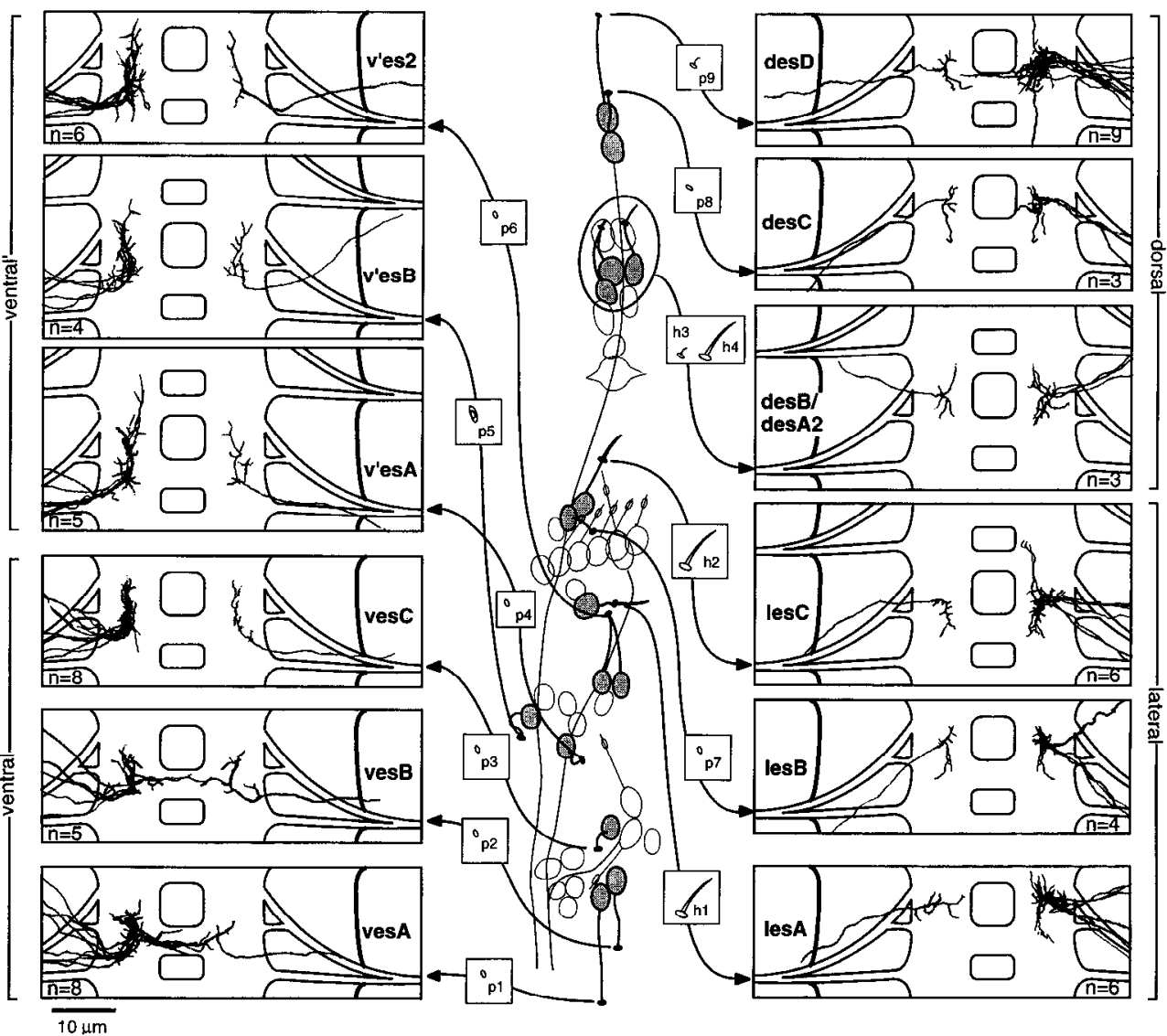




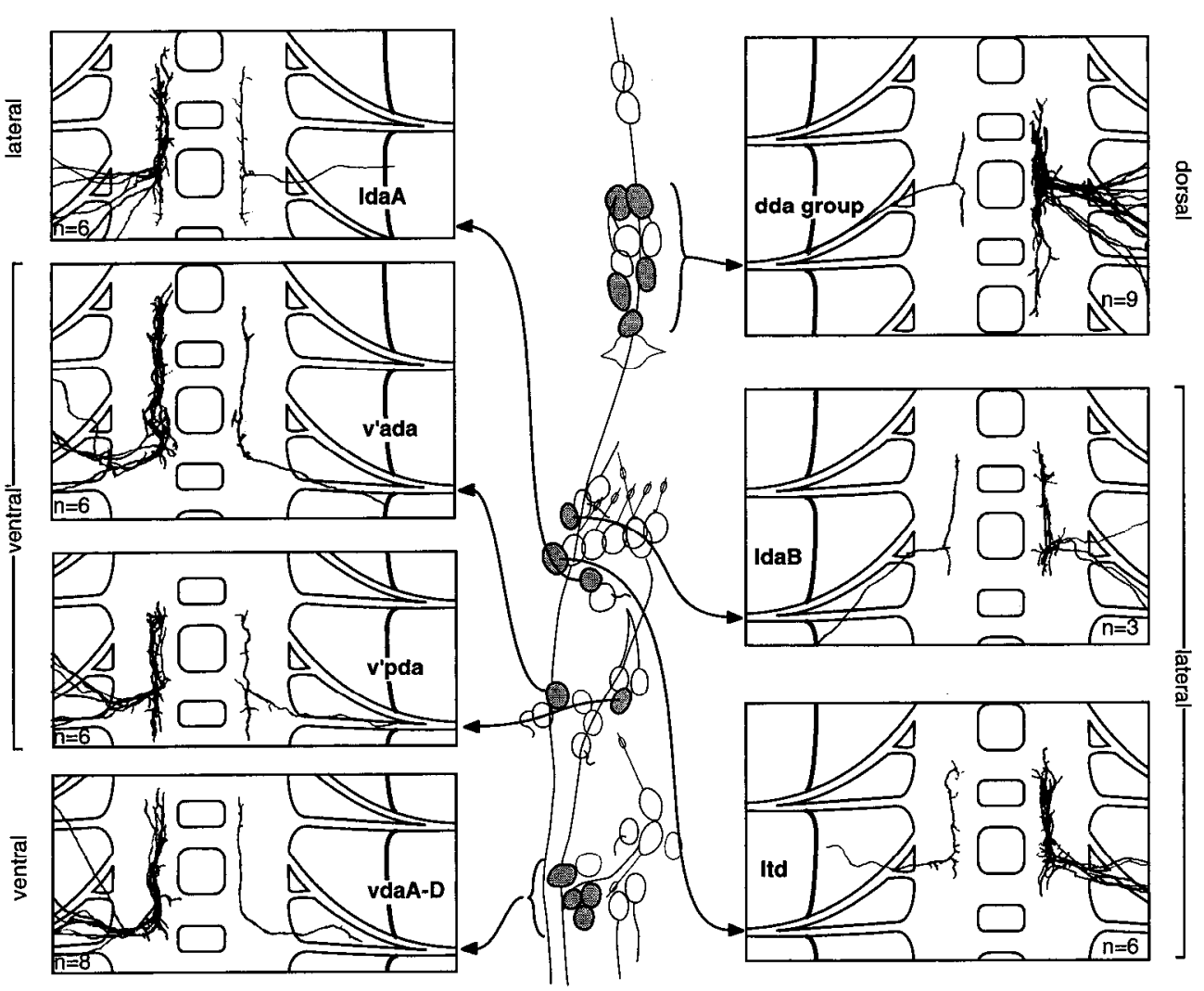

Figure 6. The central axonal projections of multiple dendrite (md) sensory neurons in the abdominal segments of the embryo. The positions of the md neurons are shown in the central panel (modified from Fig. 1: dorsal is up and anterior to the left) and their axonal projections are shown in individual panels (modified from Fig. 2: anterior is $u p$ ). Within each panel, one side shows the superimposed projections of axons from different individuals. Opposite, a single representative projection is shown. The panels are arranged to reflect the dorsal-ventral location of the parent sensory neuron: the axons of the ventralmost neurons are shown in the bottom left column and the dorsalmost in the top right column. Axon projections of individuals of the vda group are very similar so they were combined. Neurons of the dda group could not be individually identified so they too were combined. Like es and ch neurons, the axons change their pathway into the CNS at the transition between the ventral' and lateral neuronal locations. to desA2 and desB, while the axonal processes of desD show a tendency to approach or cross the midline: two out of nine axons stained have contralateral processes in the anterior commissure.

\section{md neurons}

Bodmer and Jan (1987) subdivided the multiple dendrite neurons according to their dendrite morphology or their association with trachea: da neurons have extensive dendritic arborizations beneath the hypodermis, bd neurons have bipolar dendrites, and td neurons innervate trachea. The axonal projections of these neurons conform to this subdivision, with the following exceptions: one of the td neurons has a da-like projection, and one of the da neurons (vpda) has an atypical da projection. The bd neurons have distinctive axon projections which differ from the other md neurons.

Dendritic arbor ( $m d-d a$ ). The most obvious characteristic of md-da axons is their relatively long, unbranched projection along the rostrocaudal axis (Fig. 6). Most, although not all, extend much further anteriorly than posteriorly, usually into the next anterior neuromere (Fig. 6). They show much less subbranching than either the es or ch neurons and occupy a different fascicle to the ch neurons (Fig. 3). Their positions within the connective were quantified using the method described above, resulting in a mean ML index of $0.67 \pm 0.09(n=34)$. A oneway analysis of variance reveals that the difference between the ch and the md-da ML indices is significant $(F=148.81$ on 3, $97 \mathrm{df} ; P \ll 0.001$; proportions arcsine transformed to stabilize variance). Their medial position in relation to the ch neurons was also confirmed by staining both types of neurons in the same or adjacent hemisegments of individual embryos (data not shown).

The anterior extent of the central processes varies between individual da neurons, although the same neuron in different individuals tends to have the same axonal length. The axonal projections of neurons $v d a \Lambda-D$, whose somata lie together at the periphery, are similar and are shown together in Figure 6. $\mathrm{v}$ 'pda, ldaA, Itd, and ldaB have posterior running processes although they are much shorter than the anterior process. The axonal processes of the five or six md neurons in the dorsal cluster, termed the dda group, run anteriorly or posteriorly within the same fascicle occupied by other md-da neurons (Fig. 6). The axon growth direction could not be related to a specific neuron because the dda neurons were difficult to distinguish as individuals for staining. In several cases, an axon from a neuron in this group was found to have a dorsal projection. However, since these neurons could not be identified as individuals, it is unclear whether this represents a single neuron with a distinctive nonda morphology.

The axonal projection of the vpda neuron (Fig. 7) is different than that of the other neurons of the da group. The axon enters the CNS through the posterior fascicle, typical for SOs in its location; however, the main process runs posteriorly in the longitudinal connective and subbranches run dorsally at the anterior and posterior ends of this main process. Simultaneous staining of vpda and a ventral md-da neuron show that vpda's main longitudinal axonal process runs in the same medial fascicle as the md-da axons (Fig. 8A).

Tracheal dendrites ( $m d-t d$ ). Three trachea-associated sensory neurons in each abdominal hemisegment were identified by Bodmer and Ian (1987). The Itd neuron lies deepest beneath the epidermis of any of the SOs in A1-7. The morphology of its central axonal process is typical of md-da neurons (Fig. 6). It has a mean ML index of $0.69 \pm 0.06(n=8)$; not significantly different than the md-da neurons $(0.67)$, but significantly different than the ch neurons $(0.48)$ and the other tracheal dendrite neurons (0.22) ( $P<0.05$; Tukey's multiple comparison). 


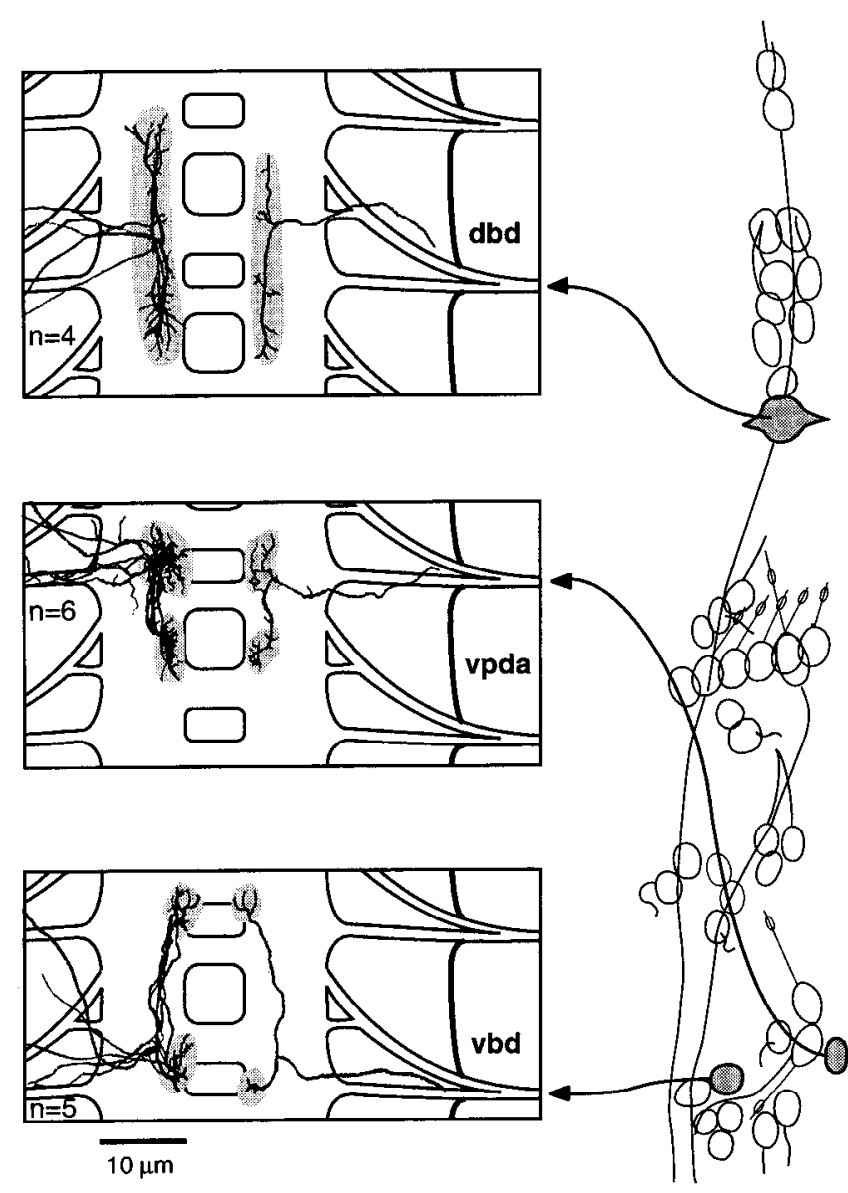

Figure 7. The central axonal projections of the bipolar dendrite md neurons and the vpda neuron in the abdominal segments of the embryo. The locations of the neurons are shown in the central panel (modified from Fig. 1: dorsal is $u p$ and anterior to the left) and the axonal projections are shown in individual panels (modified from Fig. 2: anterior is $u p$ ). Within each panel, the projections of axons from different individuals are shown on the left. On the right, a single representative projection is shown. These axons have all or part of their central processes in a dorsal layer within the CNS, highlighted here with stippling.

The axonal morphology of the two remaining td neurons, $\mathrm{v}^{\prime} \mathrm{td} 2$, is the most extensive and unusual of all sensory neurons in A1-7. The axons of the two adjacent neurons in each hemisegment follow almost identical paths in the CNS. Irrespective of their segmental origin, the axons extend anteriorly in a very lateral fascicle to the $\mathrm{T} 3$ neuromere where they turn medially and dorsally to enter a dorsal-medial longitudinal fascicle (Fig. 9). Thus, alone among sensory neurons in A1-7, they exhibit segment-specific axon projections. The lateral fascicle followed in the longitudinal connective has an ML index of $0.22 \pm 0.05$, significantly different from any other neuron type. The $v^{\prime} t d 2$ axonal projections develop relatively late in embryogenesis. The latest these neurons were successfully stained was at stage 17, just before the trachea fill with air before hatching. At this stage, some of the more posterior axons have still not reached the T3 region and, of those that have, the anterior extent within the dorsomedial fascicle is variable. It is believed that this variability is a consequence of the late onset of axon growth.

Bipolar dendrites $(m d-b d)$. There are two bipolar dendrite sensory ncurons per scgment in A1-7-one dorsal and one ventral. We believe that a third bipolar neuron (lbd) described by Bod- mer and Jan (1987) is in fact a glial cell associated with axons in the segmental border nerve; a cell in this location stains with anti-HRP, a general neuronal antibody (preparations provided by E. Ball), but not monoclonal antibody $22 \mathrm{Cl0}$, which is specific to sensory neurons (Fujita et al., 1982). Intracellular staining reveals it has no connection with the CNS. A long process extends dorsally toward the heart and a shorter process extends ventrally toward, but does not reach, the CNS. A cell in the same apparent position is stained by a glia-specific marker (Nelson and Laughon, 1993).

The soma of the ventral bipolar dendrite neuron (we have called it vbd) lies adjacent to the vdaD soma (Fig. 1). Its axonal projection is very different from the md, ch, and es neurons in that it has dorsally directed processes. The axon enters the CNS through the posterior fascicle and bifurcates into an anterior and a shorter, posterior process (Figs. $2 D, 7$ ). The dorsally directed branching occurs at the anterior and posterior ends of the main process, forming a plexus of fine branches. The main longitudinal branch is slightly greater than one segment in length, thus vbd axons from adjacent hemisegments overlap at the dorsal plexus in each hemineuromere. The anterior dorsal processes of vpda's axon project into the same dorsal plexus as vbd, shown by simultaneous staining of both neurons (Fig. $8 B$ ). Simultaneous staining of the vbd neuron and an adjacent md-da neuron of the vmd group shows that the main longitudinal branch of vbd's axon runs in the same fascicle as the md-da neurons (data not shown).

The dbd neuron is the largest sensory soma in A1-7. Its axon enters the longitudinal connective in a dorsal plane and extends rostrocaudally in a dorsal fascicle. In contrast, the axons of all other sensory neurons are restricted to the ventral layer of the connectives or, like vpda and vbd, send dorsal processes from their main longitudinal branch in this ventral fascicle. The main longitudinal branch of dbd is greater than one neuromere in length, therefore the terminal processes of dbd neurons of adjacent segments overlap in the dorsal CNS (Fig. 2D). Simultaneous staining of vbd and dbd in the same hemisegment shows that the dorsal plexus of vbd in the longitudinal connective is slightly closer to the midline than the fascicle occupied by the dbd axon (data not shown).

At the periphery, the bipolar dendrites of dbd span almost a full segment's length (Fig. 2D). The soma and dendrites are closely associated with an adjacent glial cell that forms a ligamentous structure stretched between segment borders. Glial processes also enwrap the intersegmental nerve dorsal and ventral to the glial cell soma.

\section{Discussion}

\section{Neuronal projections are type specific}

Our results show that most sensory neurons in A1-7 fall into three major categories with common axon projection attributes-the es, ch, and md-da groups-and the remaining md neurons fall into a fourth, heterogeneous category. This axonbased classification largely corresponds to classes defined solely on neuronal morphology at the periphery (Bodmer and Jan, 1987), but with some exceptions.

\section{es axons show evidence of a somatotopic arrangement}

The es neurons of the Drosophila embryo are somatotopically mapped into the CNS, a common feature of tactile neurons in insects. The axons from most ventral es organs project most posteriorly and often cross the midline, while the more lateral 

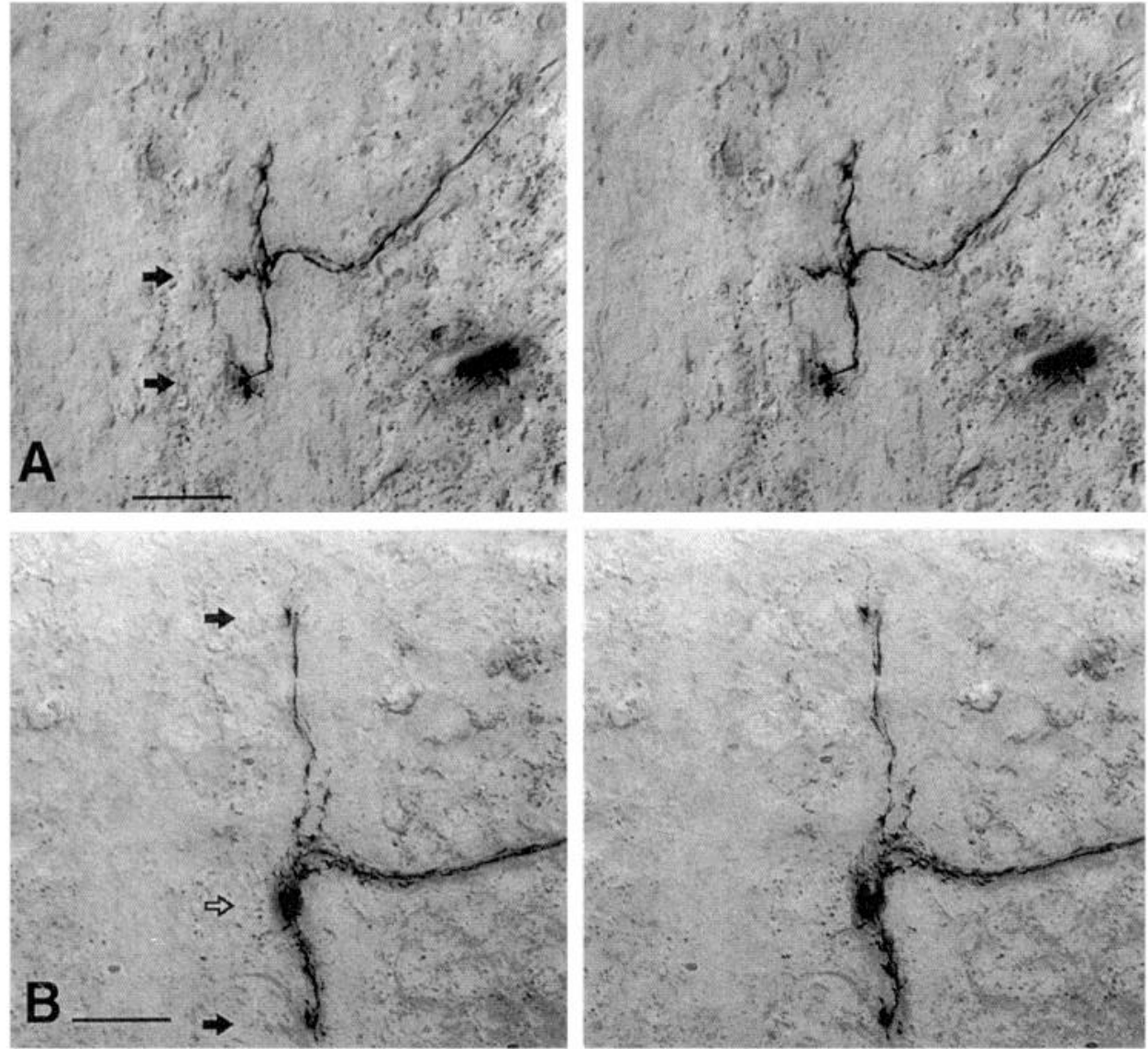

Figure 8. Stereopair views of the central axonal projections of md neuron axons. Anterior is up. A, The vpda neuron and a ventral md neuron are stained in the same hemisegment. The principle longitudinal axonal branch of both lies in the same fascicle within the CNS. The vpda neuron has dorsal-projecting terminal processes arising from this branch, indicated by arrows. $B$, The vpda neuron and vbd neuron stained in the same hemisegment showing physical overlap of the dorsal axonal processes of both neurons. The bottommost solid arrow indicates the posterior dorsal processes of vpda, the topmost solid arrow indicates the anterior dorsal processes of vbd (compare with vpda and vbd axonal processes shown in Fig. 7). The open arrow indicates the region of overlap of the anterior dorsal processes of vpda with the posterior dorsal processes of vbd. Scale bar; $100 \mu \mathrm{m}$. es organs project more anteriorly and lack medial processes. The dorsal neurons largely overlap with axons of the lateral group and do not project into a more anterior region. However, these dorsal neurons project close to and cross the midline within the same commissure as the ventral es axons. These two attributes-
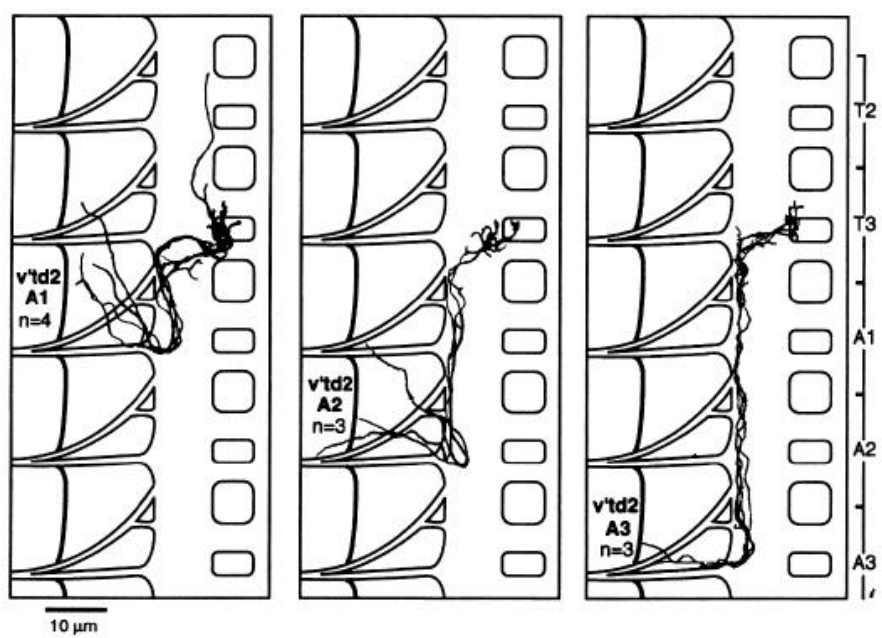

Figure 9. The central axonal projections of the v'td 2 neurons are segment specific. These neurons project anteriorly in a lateral tract into the third thoracic (T3) neuromere where they cross toward the midline and, in some cases, proceed further anteriorly. The panels show neurons from the first $(A I)$, second $(A 2)$, and third $(A 3)$ abdominal segments. Drawings of the axonal projections from neurons in several individuals were superimposed. an overall axonal distribution which shifts within the neuromere in accord with the position of the cell body in the body wall, and a tendency for sensory neurons located near the dorsal and ventral midline to have contralateral axonal processes-have been reported frequently in insects, especially for tactile neurons (Pflüger et al., 1981; Johnson and Murphey, 1985; Levine et al., 1985).

The es organs of the Drosophila embryo are most like the tactile hairs of mature insects, based on their morphology and ontogeny (Hartenstein, 1988). The es projection region may be the embryonic equivalent of the ventral association center (VAC), an anteroventral projection region for tactile afferents that is a highly conserved feature of the architecture of the insect CNS, including adult Drosophila (Johnson and Murphey, 1985; Kent and Levine, 1988; Pflüger et al., 1988; Murphey et al., 1989b; Merritt and Murphey, 1992). In larger insects, where finely graded distinctions in axon projections are visible at the light microscope level, the VAC has been shown to be two layered, with the axons from dorsally located tactile SOs projecting into a topmost and slightly more anterior layer (anterior VAC) and the ventral SOs into a ventralmost layer (ventral VAC) (Johnson and Murphey, 1985; Kent and Levine, 1988; Pflüger et al., 1988). The ventral and dorsal group axons in the Drosophila embryo may be similarly layered within the CNS.

Within each segment, the somatotopic projection of the es organs is independent of the point of axon entry into the CNS. All SOs of the $v$ and $v^{\prime}$ regions enter the CNS through the posterior fascicle, and all of the lateral and dorsal neurons enter through the anterior fascicle: thus mixed types of axons enter 
through each fascicle. The graded somatotopy of es neurons is maintained within the CNS despite the shift of axon entry of the dorsal and ventral neurons. The same phenomenon occurs in Manduca; the somatotopy of es axons is independent of the point of entry of the tactile axons into the CNS through either an anterior or posterior nerve (Levine et al., 1985).

Topographic mapping of sensory input is a fundamental property of nervous system organization. Given the extensive knowledge of how segment polarity and positional information is established in Drosophila, the embryo may provide the avenue for investigating the genetic basis of how neuronal position is translated into central axonal projections.

\section{How many subtypes of es organs?}

es organs are not a homogeneous group. They may have cuticular hairs or papillae or may be mono- or multi-innervated. Do axon projections reflect the subtypes of es organs?

Two es organs per segment in $\Lambda 1-7$ are multi-innervated: des 2 and v'es2. Their stimulus specificity is unknown but such multiply innervated neurons are often gustatory or thermohygroreceptive (Steinbrecht, 1984; Hartenstein, 1988). If the neurons innervating this organ in the abdomen are of a different type than the other es neurons, this is not reflected in their axonal projections at the level of resolution provided by this study.

Also, there appear to be no qualitative differences between the projections of es neurons innervating hairs and those innervating papillae. The papillae resemble campaniform sensilla (CS) in other insects. Typically, insect CS neurons have fundamentally different axon projections to tactile neurons: tactile neurons project into ventral neuropil in the CNS while CS neurons normally project into a more dorsal layer in common with proprioceptive neurons (Murphey et al., 1985; Pflüger et al., 1988; Merritt and Murphey, 1992). These classical proprioceptive $C S$ are usually found in clusters near joints where they monitor shear stresses in the cuticle. However, another type of CS has been described on the cerci of crickets and wings of Drosophila (Merritt and Murphey, 1992; Killian et al., 1993). Unlike classical CS, these SOs are distributed diffusely, they are not associated with joints, and they have axonal projections typical of tactile neurons. It has been argued that they represent a phylogenetically recent reduction of the external cuticular apparatus of tactile hairs to resemble proprioceptive CS (Killian et al., 1993). The CS of the Drosophila larva may be of this type rather than true CS. This is not surprising, as classical CS are rare on the body segments of adult flies, being most common on the appendages (Gnatzy et al., 1987), and therefore unlikely to be found on the abdominal segments of the larva. In addition, the soft body of the larva may render the cuticular stress detection function of classic CS ineffectual. A truncation of hair length in a subset of the abdominal tactile sense organs to produce a papilla-like shape may have occurred as an adaptation to specific aspects of the specialized dipteran larval environment.

Higashijima et al. (1992) also concluded that the larval papillae represent truncated hairs rather than a different class of SO. The papilla- or hair-like shape of the cuticular structure secreted by the trichogen cell is closely related to the level of $\mathrm{BarH1}$ and $\mathrm{BarH} 2$ expression in the SO, with structural intermediates being observed in Bar mulant embryos. Thus the basiconic and hair-like SOs belong to the same category. ch neuron projections are conserved in ontogeny and phylogeny

All ch neurons of A1-7 project into a common central region of the longitudinal connective within each neuromere. They have long processes along the rostrocaudal axis and there is no tendency for axons to approach or cross the midline, nor is there is an obvious somatotopic projection.

The projection of ch axons into a discrete region of the CNS appears to be a feature of ch neuron identity which is conserved through development and evolution, just as a specific projection region of tactile neurons is conserved from embryo to adult and across a phylngenetic range of insects. Recently, it has been shown that the lch5 neurons of Drosophila are homologous to the pleural ch organs of the grasshopper embryo (Meier et al., 1991). In the grasshopper, the ch organs persist through to the adult, where their axonal processes in the CNS have been stained and found to extend along a recognizable longitudinal tract, the ventral intermediate tract (VIT) (Boyan, 1993). It follows that this tract may be homologous to the medial fascicle occupied by ch axons in the Drosophila embryo. The lch5 organs are lost in the transition to the adult in Drosophila; the entire larval PNS degenerates during the pupal phase and the CNS is extensively remodeled. However, homologs of the VIT have been identified in the CNS of adult flies (Merritt and Murphey, 1992). We predict that ch organs of the body wall of the adult project to this region.

md neurons display a variety of axon projections and possible sensory functions

The multiple dendrite neurons form the third distinctive type of SO. Relatively little is known of the development, fine structure, or function of these SOs (Ghysen and Dambly Chaudière, 1992). Physiological analyses have shown that they are primarily mechanoreceptive. Their concentration around articular joints in hardbodied insects suggests that they may generally have a proprioceptive function (Finlayson, 1976; Wright, 1976); however, in the soft-bodied Drosophila larva, the dendrites of the md-da neurons form a widespread network and may function as receptors for punctate stimuli (Bodmer and Jan, 1987). The md-da neurons of A1-7 project into a common region of the longitudinal connective within each neuromere. Like ch axons, they have long processes along the rostrocaudal axis and there is no tendency for axons to approach or cross the midline; nor is there is an obvious somatotopic projection.

Through the lack of additional characters, a categorization of md neurons into three groups, md-da, md-bd, and md-td, has been based primarily on their dendrite morphology-the md-da neurons have diffuse dendritic arbors, the bd neurons have bipolar dendrites - and secondarily on the association of dendrites with trachea in the td neurons (Bodmer and Jan, 1987). Our knowledge of their axon projections leads to a reassessment of this classification. The md-da neurons form a discrete group based on axon projections, which includes 13 of the 19 md neurons of a hemisegment. We have added one neuron to the mdda group-the tracheal neuron, Itd, has an axon projection indistinguishable from the md-da neurons-and two neurons have been eliminated because they do not show this type of projection: vpda has a conspicuous dorsal-running component to the axon projection, and we suspect that one neuron in the dorsal cluster may have the same characteristic.

These two neurons and the remaining md neurons have het- 
erogeneous axon projections when compared to the md-da neulrons. The two remaining trachea-associated neurons $\left(v^{\prime} t d 2\right)$ have distinctive axonal projections which bear no similarity to ltd's axon projection. Thus the association of dendrites with particular structures such as trachea does not appear to be a reliable indicator of axonal projection. These paired neurons are unusual: they are the only neurons in A1-7 whose axonal projection depends on the identity of the abdominal segment in which they lie. The axons extend anteriorly in a lateral fascicle as far as the metatoracic neuromere, where they switch to a dorsomedial fascicle. Their function is unknown. They may monitor aspects of tracheal physiology (Bodmer and Jan, 1987). On the other hand, their attachment to trachea may be incidental: it could merely be a convenient substrate for the attachment of stretch-receptive dendrites.

The two bipolar dendrite neurons have distinctive, but dissimilar, axon projections: dbd's axon enters the CNS dorsally while vbd's axon runs for some length in the md fascicle before projecting dorsally. The vpda axon also does this, suggesting that vpda and vbd may have more in common with the md-da group than do v'td2 and dbd.

The bipolar dendrite neurons are ideally placed to function as proprioceptors: a sensitivity to longitudinal stretching would provide feedback on segmental length changes during locomotory contraction and relaxation. The axon terminals of both vbd and dbd overlap with their counterparts from adjacent segments, forming a linked chain of sensory axons along the abdominal neuromeres, leading us to speculate that the regions of overlap could represent integrative regions for processing proprioceptive input from adjacent segments. Based on physiological experiments in larger insects, sensory neurons which project into dor sal CNS, as do dbd, vbd, and vpda in the embryo, are candidates for forming monosynaptic connections with motor neurons. Direct motor-sensory synapses are most common with proprioceptive sensory neurons such as campaniform sensilla, multipolar stretch receptors, or ch organs, where there is physical overlap of sensory and motor processes in the intermediate or dorsal neuropil (Pearson et al., 1976; Burrows and Pflüger, 1988; Laurent and Hustert, 1988; Burrows, 1992). For example, the wing hinge stretch receptor of grasshoppers is an md neuron with extensive arborizations in the dorsal CNS, where it makes monosynaptic contact with flight motor neurons (Burrows, 1975). We plan to assess the degree of overlap between sensory axons and individually identifiable motor neurons in the CNS (Sink and Whitington, 1991).

\section{A categorization based on axonal projections correlates with proneural gene specificity}

The 13 md-da neurons per hemisegment form a homogeneous group based on both axon and dendrite morphology. v'td2, vpda, vbd, dbd, and perhaps another dorsal md neuron comprise a heterogeneous group with relatively few shared features. Remarkably, this axon-based categorization of the md neurons corresponds precisely with the class of proncural gene that initiates the neurogenic pathway of the individual SOs (Table 1). The two categories of proneural genes involved in embryonic PNS determination are the achaete-scute complex (ASC) and the atonal gene: ASC genes specify the es organs and a subset of md neurons (Dambly-Chaudière and Ghysen, 1987; Jarman et al., 1993); the atonal gene specifies all ch organs and most of the remaining subset of Ind neurons (Jarman et al., 1993). The subset of md neurons specified by ASC corresponds exactly with
Table 1. The correlation between proncural genes, types of sensory neurons, and their axonal projections

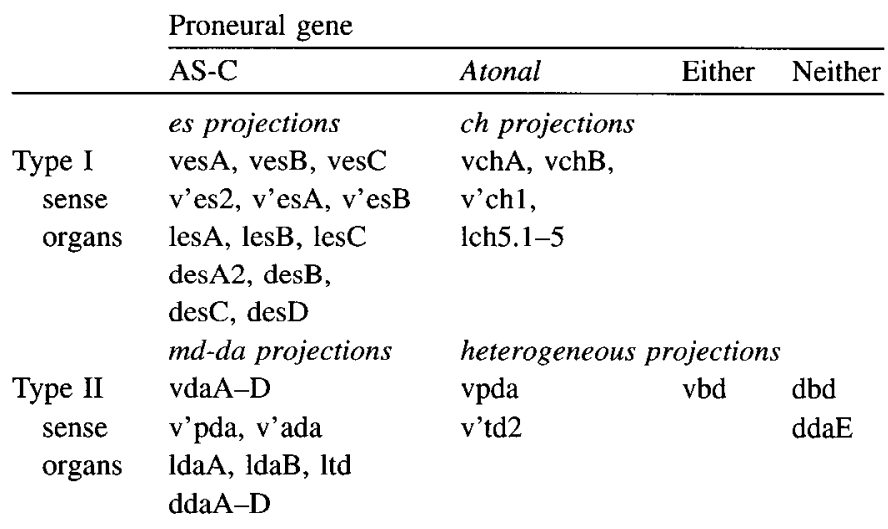

The sensory neurons of an abdominal segment are divided according to their requirement for particular proneural genes-genes of the achaete-scute complex (AS-C), the atonal gene, both, or either-based on their absence in mutants for these particular genes (Dambly-Chaudière and Ghysen, 1987; Jarman et al, 1993). They are further divided into type I and II neurons according to their dendritic characteristics. Three of the resulting subgroups have common axonal projection types (see text). The remaining subgroup-type II neurons specified by atonal, either atonal or AS-C, or neither proneural gene-includes md neurons with individual, characteristic projections.

our md-da class, which is based exclusively on similar axon projection attributes. The remaining md organs, those with heterogeneous axon projections, are specified by atonal, either atonal or AS-C, or neither (there is some uncertainty in md neuron association with particular proneural genes because of individual variation in the neurons deleted in the genetic deficiencies and the difficulty in identifying individual neurons where the organization of the PNS is disrupted: Dambly-Chaudière and Ghysen, 1987; Jarman et al., 1993). The same categorization applies to the type I SOs. The es neurons have common axon projection features and all es organs are specified by genes of the ASC. Similarly, all of the ch neurons project into a common region and all are specified by atonal. These correlations suggest that the nature of the proneural gene may have a direct influence on the identity of the resulting $\mathrm{SO}$ as revealed by the axonal projection of the sensory neuron.

An analysis of ectopic proneural gene expression has led to the same conclusion, at least for the type I SOs (Jan and Jan, 1993; Jarman et al., 1993). atonal is both necessary and sufficient for ch organ formation based on the loss-of-function phenotype and the formation of extra ch organs with ectopic atonal expression (Jarman et al., 1993). The effect of ectopic ASC expression has not been specifically investigated in the embryo; however, scute overexpression in the imaginal discs produces additional es organs in the adult (Rodríguez et al., 1990).

The specification of mixed SOs of types I and II by the proneural genes remains a problem. If the proneural genes specify neuronal identity, how can each simultancously specify two different types, namely es together with md-da, and ch with md? Addressing this problem, Ghysen and Dambly-Chaudière (1992) speculate that there could be a common origin of both precursors, or some form of induction of type II precursors by a type I precursor. This is based on (1) the physical proximity of many md-da neurons and es organs and (2) the removal of both an es organ and the adjacent md neuron in ASC genetic deletions. However, this mechanism would not explain why all es and ch 


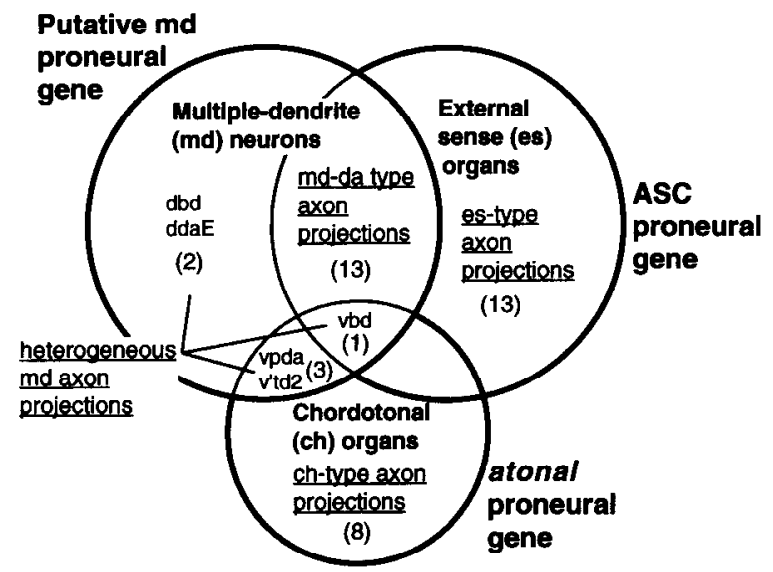

Figure 10. The hypothetical relationship between the known proneural genes-genes of the achaete-scute complex and atonal-and a putative md proneural gene that specifies the multiple-dendrite neurons. Circles represent realms of proneural gene activity: sense organs specified by a single proneural gene are identified in regions of no overlap. Combined expression of proneural genes in the ectoderm, indicated by overlapping segments of circles, gives rise to sense organs of different types. The numbers of sense organs of each type present in an abdominal hemisegment are shown in parentheses. The specificity of proneural genes for particular sense organs is taken from Table 1.

organs do not have adjacent md neurons, and why some md neurons arise in clusters apart from es or ch neurons.

The mechanism whereby type I and II SOPs are differentiated from among ectodermal cells expressing each of the proneural genes is significant because this represents a fundamental choice in neuronal identity: the choice between type I SOs with support cells and ciliated, monopolar neurons, and type II SOs with no support cells and nonciliated neurons. This may represent a phylogenetically ancient division of sensory type as both types of SOs are found in crustaceans, centipedes, millipedes, and arachnids, in addition to insects (Finlayson, 1968, 1976; Wright, 1976).

We propose that an additional proneural gene is involved in the specification of md precursors. The subtype of md neuron could then be determined by whether this putative proneural gene is expressed alone in a proneural patch or in combination with atonal or ASC. The possible relationship between expression of this putative proneural gene and known proneural genes is shown in diagrammatic form in Figure 10, based on the simplest interpretation in which a single such gene is responsible for specification of all md organs: it remains possible that a gene complex, like the achaete-scute complex, or a number of unrelated genes could be involved. This model, which calls upon combinatorial expression of proneural genes to specify the variety of SO types, accommodates the known loss-of-function phenotypes of ASC and atonal: in ASC deletions all md-da and es organs are lost, and in atonal deletions, three md neurons and all ch organs are lost. The vbd neuron is present in deletions of either ASC or atonal although it is absent in the double mutation (Jarman et al., 1993), suggesting that it can be specified by either ASC or atonal. The putative md proneural gene appears to be solely responsible for only two neurons per hemisegment, dbd and ddaE, unlike ASC and atonal, both of which alone specify a considerable subset of SOs: 13 es and eight ch organs, respectively. This hypothesis may account for the physical proximity of type I and type II neurons, noted by Ghysen and Dambly-Chaudière (1992): if one of the overlapping proneural genes is expressed in relatively small patches, then different types of SOPs will arise close together. As yet unknown proneural genes have been called upon to explain the specification of the two md neurons that develop in the absence of both AS-C and atonal (Jarman et al., 1993). The mechanism suggested here may explain the specification of a diversity of neuronal types by interaction of a small number of proneural genes.

\section{References}

Bacon J, Murphey R (1984) Receptive fields of cricket giant interneurones are related to their dendritic structure. J Physiol (Lond) 352: 601-623.

Bodmer R, Jan YN (1987) Morphological differentiation of the embryonic peripheral neurons in Drosophila. Roux's Arch Dev Biol 196:69-77.

Bodmer R, Barbel S, Sheperd S, Jack JW, Jan LY, Jan YN (1987) Transformation of sensory organs by mutations of the cut locus of D. melanogaster. Cell 51:293-307.

Bodmer R, Caretto R, Jan YN (1989) Neurogenesis of the peripheral nervous system in Drosophila embryos: DNA replication patterns and cell lineages. Neuron 3:21-32.

Boyan G (1993) Another look at insect audition: the tympanic receptors as an evolutionary specialization of the chordotonal system. J Insect Physiol 39:187-200.

Burrows M (1975) Monosynaptic connexions between wing stretch receptors and flight motoneurones of the locust. J Exp Biol 62:189219.

Burrows M (1992) Local circuits for the control of leg movements in an insect. Trends Neurosci 15:226-232.

Burrows M, Pflüger HJ (1988) Positive feedback loops from proprioceptors involved in leg movements of the locust. J Comp Physiol A $163: 425-440$

Campos-Ortega J, Hartenstein V (1985) The embryonic development of Drosophila melanogaster. Berlin, Heidelberg, New York: Springer.

Dambly-Chaudière C, Ghysen A (1986) The sense organs in the Drosophila larva and their relation to the embryonic pattern of sensory neurons. Rouxs Arch Dev Biol 195:222-228.

Dambly-Chaudic̀re C, Ghysen A (1987) Independent subpatterns of sense organs require independent genes of the achaete-scute complex in Drosophila larvae. Genes Dev 1:297-306.

Dambly-Chaudière C, Jamet E, Burri M, Bopp D, Basler K, Hafen E, Dumont N, Spielmann P, Ghysen A, Noll M (1992) The paired box gene pox-neuro: a determinant of polyinnervated sense organs in Drosophila. Cell 69:159-172.

Finlayson LH (1968) Proprioceptors in the invertebrates. Symp Zool Soc Lond 23:217-249.

Finlayson L (1976) Abdominal and thoracic receptors in insects, centipedes and scorpions. In: Structure and function of proprioceptors in the invertebrates (Mill P, ed), pp 153-212. London: Chapman and Hall.

Fujita SC, Zipursky SL, Benzer S, Ferrus A, Shotwell SL (1982) Monoclonal antibodics against the Drosophila nervous system. Proc Natl Acad Sci USA 79:7929-7933.

Ghysen A (1980) The projection of sensory neurons in the central nervous system of Drosophila: choice of the appropriate pathway. Dev Biol 78:521-541.

Ghysen A, Dambly-Chaudière C (1988) From DNA to form: the achaete-scute complex. Genes Dev 2:495-501.

Ghysen A, Dambly-Chaudière C (1992) Development of the peripheral nervous system in Drosophila. In: Determinants of neuronal identity (Shankland M, Macagno ER, ed), pp 225-292. San Diego: Academic.

Ghysen A, Dambly-Chaudière C (1993) The specification of sensory neuron identity in Drosophila. Bioessays 15:293-298.

Ghysen A, Dambly-Chaudière C, Aceves E, Jan LY, Jan YN (1986) Sensory neurons and peripheral pathways in Drosophila embryos. Rouxs Arch Dev Biol 195:281-289.

Ghysen A, Dambly-Chaudière C, Jan LY, Jan YN (1993) Cell interactions and gene interactions in peripheral neurogenesis. Genes Dev 7:723-733.

Giniger E, Wells W, Jan LY, Jan YN (1993) Tracing neurons with a kinesin- $\beta$-galactosidase fusion protein. Rouxs Arch Dev Biol 202: $112-122$.

Gnatzy W, Grünert U, Bender M (1987) Campaniform sensilla of Cal- 
liphora vicina (Insecta, Diptera). I. Topography. Zoomorphology 106: 312-319.

Hartenstein V (1988) Development of Drosophila larval sensory organs: spatiotemporal pattern of sensory neurones, peripheral axonal pathways and sensilla differentiation. Development 102:869-886.

Higashijima S, Michiue T, Emori Y, Saigo K (1992) Subtype determination of Drosophila embryonic external sensory organs by redundant homeo box genes BarH1 and BarH2. Genes Dev 6:10051018 .

Jan YN, Jan LY (1990) Genes required for specifying cell fates in Drosophila embryonic sensory nervous system. Trends Neurosci 13: 493-.

Jan YN, Jan LY (1993) HLH proteins, fly neurogenesis, and vertebrate myogenesis. Cell 75:827-830.

Jarman AP, Grau Y, Jan LY, Jan YN (1993) atonal is a proneural gene that directs chordotonal organ formation in the Drosophila peripheral nervous system. Cell 73:1307-1321.

Johnson SE, Murphey RK (1985) The afferent projection of mesothoracic bristle hairs in the cricket Acheta domesticus. J Comp Physiol A 156:369-379.

Kent KS, Levine RB (1988) Neural control of leg movements in a metamorphic insect: sensory and motor elements of the larval thoracic legs in Manduca sexta. J Comp Neurol 271:559-576.

Killian KA, Merritt DJ, Murphey RK (1993) Transplantation of neurons reveals processing areas and rules for synaptic connectivity in the cricket nervous system. J Neurobiol 24:1187-1206.

Laurent G, Hustert R (1988) Motor neuronal receptive fields delimit patterns of motor activity during locomotion of the locust. J Neurosei 8:4349-4366.

Levine RB, Pak C, Linn D (1985) The structure, function and metamorphic reorganization of somatotopically projecting sensory neurons in Manduca sexta larvae. J Comp Physiol A 157:1-13.

Meier T, Chabaud F, Reichert H (1991) Homologous patterns in the embryonic development of the peripheral nervous system in the grasshopper Schistocerca gregaria and the fly Drosophila melanogaster. Development 112:241-253.

Merritt DJ, Murphey RK (1992) Projections of leg proprioceptors within the CNS of the fly Phormia in relation to the generalized insect ganglion. J Comp Neurol 322:16-34.

Merrill DJ, Hawken A, Whitington PM (1993) The role of the cut gene in the specification of central projections by sensory axons in Drosophila. Neuron 10:741-752.

Murphey RK, Bacon JP, Johnson SE (1985) Ectopic neurons and the organization of insect sensory systems. J Comp Physiol A 156:381389.

Murphey RK, Possidente DR, Vandervorst P, Ghysen A (1989a) Com- partments and topography of leg afferent projections in Drosophila. J Neurosci 9:3209-3217.

Murphey RK, Possidente DR, Pollack G, Merritt DJ (1989b) Modalityspecific axonal projections in the CNS of the flies Phormia and Drosophila. J Comp Neurol 290:185-200.

Nelson HB, Laughon A (1993) Drosophila glial architecture and development: analysis using a collection of new cell-specific markers. Rouxs Arch Dev Biol 202:341-354.

Newland PL (1991) Morphology and somatotopic organisation of the central projections of afferents from tactile hairs on the hind leg of the locust. J Comp Neurol 312:493-508.

Nottebohm E, Dambly-Chaudière C, Ghysen A (1992) Connectivity of chemosensory neurons is controlled by the gene poxn in Drosophila. Nature 359:829-832.

Pearson KG, Wong RKS, Fourtner CR (1976) Connexions between hair-plate afferents and motoneurones in the cockroach leg. $J$ Exp Biol 64:251-266.

Peterson BA, Weeks JC (1988) Somatotopic mapping of sensory neurons innervating mechanosensory hairs on the larval prolegs of Manduca sexta. J Comp Neurol 275:128-144.

Pflüger HJ, Bräunig P, Hustert R (1981) Distribution and specific central projections of mechanoreceptors in the thorax and proximal leg joints of locusts. II. The external mechanoreceptors: hair plates and tactile hairs. Cell Tissue Res 216:79-96.

Pflüger HJ, Bräunig P, Hustert R (1988) The organization of mechanosensory neuropiles in locust thoracic ganglia. Phil Trans R Soc Lond [Biol] 321:1-26.

Rodríguez I, Hernández R, Modolell J, Ruiz-Gómez M (1990) Competence to develop sensory organs is temporally and spatially regulated in Drosophila epidermal primordia. EMBO J 9:3583-3592.

Sandell JH, Masland RH (1988) Photoconversion of some fluorescent markers to a diaminobenzidine product. J Histochem Cytochem 36: $555-559$.

Sink H, Whitington PM (1991) Location and connectivity of abdominal motoneurons in the embryo and larva of Drosophila melanogaster. J Neurubiol 22:298-311.

Steinbrecht R (1984) Arthropoda: chemo-, hygro-, and thermoreceptors. In: Biology of the integument (Bereiter-Hahn J, Matoltsy A, Richards K, eds), pp 523-553. Berlin: Springer.

Vaessin H, Caudy M, Bier E, Jan LY, Jan YN (1990) Role of helixloop-helix proteins in Drosophila neurogenesis. Cold Spring Harbor Symp Quant Biol 55:239-245.

Wieschaus E, Nüsslein-Volhard C (1986) Looking at embryos. In: Drosophila: a practical approach (Roberts DB, ed), pp 199-227. Oxford: IRL.

Wright BR (1976) Limb and wing receptors in insects, chelicerates and myriapods. In: Structure and function of proprioceptors in the invertebrates (Mill P, ed), pp 323-386. London: Chapman and Hall. 